\title{
ALMA as the ideal probe of the solar chromosphere
}

\author{
Maria A. Loukitcheva • Sami K. Solanki • \\ Stephen White
}

Received: 31 January 2007 / Accepted: 26 July 2007 / Published online: 9 September 2007

(C) Springer Science+Business Media B.V. 2007

\begin{abstract}
The very nature of the solar chromosphere, its structuring and dynamics, remains far from being properly understood, in spite of intensive research. Here we point out the potential of chromospheric observations at millimeter wavelengths to resolve this long-standing problem. Computations carried out with a sophisticated dynamic model of the solar chromosphere due to Carlsson and Stein demonstrate that millimeter emission is extremely sensitive to dynamic processes in the chromosphere and the appropriate wavelengths to look for dynamic signatures are in the range 0.8 $5.0 \mathrm{~mm}$. The model also suggests that high resolution observations at mm wavelengths, as will be provided by ALMA, will have the unique property of reacting to both the hot and the cool gas, and thus will have the potential of distinguishing between rival models of the solar atmosphere. Thus, initial results obtained from the observations of the quiet Sun at $3.5 \mathrm{~mm}$ with the BIMA array (resolution of $12^{\prime \prime}$ ) reveal significant oscillations with amplitudes of $50-150 \mathrm{~K}$ and frequencies of $1.5-8 \mathrm{mHz}$ with a tendency toward short-period oscillations in internetwork and longer periods in network regions. However higher spatial resolution, such as that provided by ALMA, is required for a clean separation between the features within the solar atmosphere and for an adequate
\end{abstract}

M.A. Loukitcheva ( $\varangle) \cdot$ S.K. Solanki

Max-Planck-Institut für Sonnensystemforschung,

37191 Katlenburg-Lindau, Germany

e-mail: lukicheva@mps.mpg.de

M.A. Loukitcheva

Astronomical Institute, St. Petersburg University,

Universitetskii pr. 28, Peterhof, 198504 St. Petersburg, Russia

S. White

Astronomy Department, University of Maryland, College Park, MD 20742, USA comparison with the output of the comprehensive dynamic simulations.

Keywords Sun · Solar chromosphere · Millimeter observations

\section{Introduction}

The chromosphere remains the least understood layer of the solar atmosphere, with the very basics of its structure being hotly debated: is it better described by the classical picture of a steady temperature rise as a function of height, with superposed weak oscillations (e.g. semi empirical models of Vernazza et al. (1981); Fontenla et al. (1990)), or does the temperature keep dropping outwards, with very hot shocks producing strong localized heating (radiation hydrodynamic simulations of Carlsson and Stein (1995, 2002), and Wedemeyer et al. (2004))? The latter concept is consistent with the IR observations of carbon monoxide, which require cool gas to be present at chromospheric heights (see, e.g. Ayres 2002).

Thus, existing models cannot provide a complete description of the solar chromosphere. Consequently nowadays two alternative pictures of the chromosphere co-exist and the role played by chromospheric dynamics in the structuring of this atmospheric layer is a subject of intense scientific debate.

One reason for conflicting models is that they are based either on atomic chromospheric lines and continua in the UV or on molecular lines in the IR, since UV observations are practically blind to cool gas in a dynamic chromosphere, while the IR observations sample only the cool part of the chromosphere. Improved and more sensitive diagnostics of 
the chromospheric structure and dynamics, that sample both the hot and the cool gas and should distinguish between the rival models, are provided by observations at millimeter wavelengths with an acceptable spatial resolution as was proposed by Loukitcheva et al. (2004). In this contribution we review the unique chromospheric observations at $3.5 \mathrm{~mm}$ with the Berkeley-Illinois-Maryland Array and the analysis of the intensity variations expected from the model of Carlsson and Stein for mm wavelengths. We postulate the requirements for mm observations with the future instruments, with emphasis on spatial and temporal resolution. Finally we discuss the prospects for chromospheric studies with ALMA.

\section{Results}

\subsection{Analysis of the BIMA observations at $3.5 \mathrm{~mm}$}

The Berkeley-Illinois-Maryland Array (BIMA) operating at a wavelength of $3.5 \mathrm{~mm}$ (frequency of $85 \mathrm{GHz}$ ) has been the only interferometer in the mm range frequently used for solar observations. The BIMA telescopes are now part of the CARMA array which will also carry out such observations. With the BIMA data obtained in the years 2003 and 2004 we have constructed two-dimensional maps of the solar chromosphere with a resolution of $12^{\prime \prime}$, which represents the highest spatial resolution achieved so far at this wavelength for non-flare solar observations. The BIMA images have led to new insights in to chromospheric structure and to the detection of spatially-resolved chromospheric oscillations at $\mathrm{mm}$ wavelengths. The details of the restoration procedure and extensive tests of the sensitivity of the BIMA data to the detection of dynamic signatures can be found in White et al. (2006).

With the currently available resolution the contrast of the brightness structures is evaluated to be up to $30 \%$ of the quiet-sun brightness at $3.5 \mathrm{~mm}$ (White et al. 2006). However, the similarity of brightness structures, derived from the $\mathrm{mm}$ images and seen in other chromospheric emissions (Fig. 1), in spite of the difference in resolution of the images (1-2" resolution of the UV images), implies that the BIMA resolution is not enough to resolve the millimeter fine structure and observations with spatial resolution much higher than $12^{\prime \prime}$ are required. A detailed analysis of the relations between the millimeter emission, magnetic field and other chromospheric diagnostics is in preparation.

In the millimeter brightness we detected intensity oscillations with typical amplitudes of $50-150 \mathrm{~K}$ in the range of periods from 120 to 700 seconds (frequency range 1.5$8 \mathrm{mHz}$ ). We found a tendency toward short period oscillations in internetwork and longer periods in network regions in the quiet Sun, which is in good agreement with the results obtained at other wavelengths. At $3 \mathrm{~mm}$ the inner parts of the chromospheric cells exhibit a behavior typical of the internetwork with the maximum of the Fourier power in the 3 -minute range, however, most of the oscillations are quasiperiodic, showing up in wave trains of finite duration lasting for typically 1-3 wave periods (see also Loukitcheva et al. 2006).

\subsection{Analysis of the CS model millimeter spectrum}

The response of the submillimeter and millimeter radiation to a time-series generated by Carlsson and Stein (CS) was computed under the assumption of thermal free-free radiation by Loukitcheva et al. (2004). The results are depicted in Fig. 2 as the excess intensity as a function of wavelength and time.

Wave periods of approximately 3 min can be clearly distinguished in the intensity at all considered wavelengths. Though the dominant frequency of the oscillations changes slightly with wavelength, for all $\mathrm{mm}$ wavelengths it lies in the range of 3 minutes. The difference from one period of time to another can be explained by the presence of merging shocks during certain time intervals. The differences in the light curves at different wavelengths are caused primarily by the difference in the formation heights of the emitted radiation. In general the amplitudes of the oscillations compared to the radiation temperature are large, in this sense $\mathrm{mm}$ wavelength radiation combines the advantages of the $\mathrm{CO}$ lines, which mainly see the cool gas, with those of atomic lines and UV continua, which mainly sample the hot gas.

On the whole, the brightness temperatures are extremely time-dependent at millimeter wavelengths, following changes in the atmospheric parameters. With increasing wavelength the amplitude of the brightness oscillations grows significantly, reaches its maximum value at $2.2 \mathrm{~mm}$ (expected to be $15 \%$ of the quiet-Sun brightness temperature), and decreases rapidly towards longer wavelengths. Thus we can identify the range $0.8-5.0 \mathrm{~mm}$ as the appropriate range of $\mathrm{mm}$ wavelengths at which one can expect the clearest signatures of dynamic effects. A careful look at the $\mathrm{mm}$ brightness spectrum as a function of time (see Fig. 2) reveals a time delay between the oscillations at long and short millimeter wavelengths. Hence, it is possible to study wave modes traveling in the chromosphere by comparing sub-mm with mm observations.

\section{Discussion}

The CS model predicts that spatially and temporally resolved observations should clearly exhibit the signatures of the strong shock waves. However, a direct comparison of the observational data products (RMS values, histogram skewness, Fourier and wavelet spectra, etc.), referring to regions 
Fig. 1 Portrait of the solar chromosphere at the center of the Sun's disk at 4 different wavelengths on May 18, 2004. From top left to bottom right: MDI longitudinal photospheric magnetogram, UV $1600 \mathrm{~A}$ image from TRACE, CaII K line center image from BBSO and BIMA image at $3.5 \mathrm{~mm}$
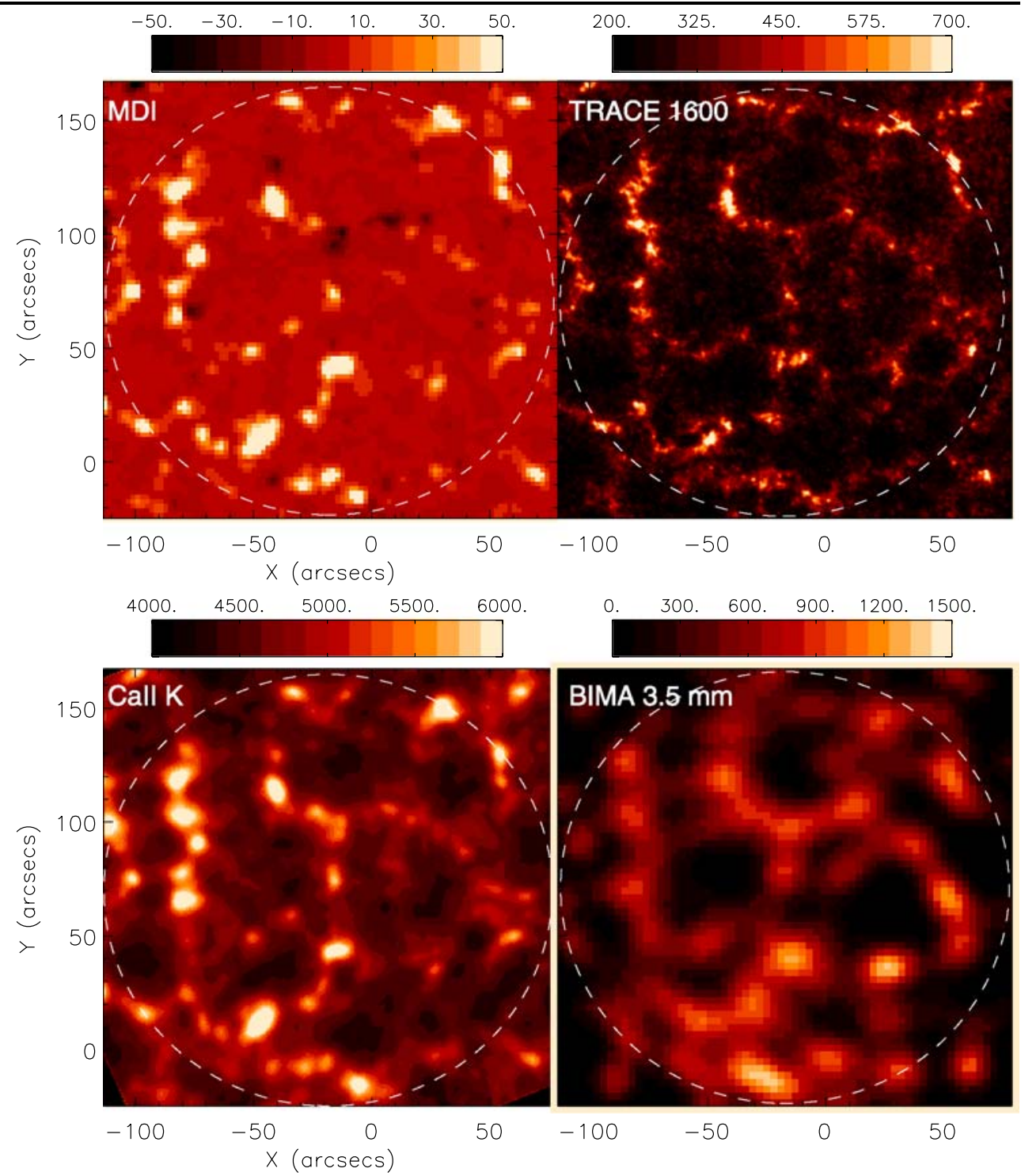

$-100$

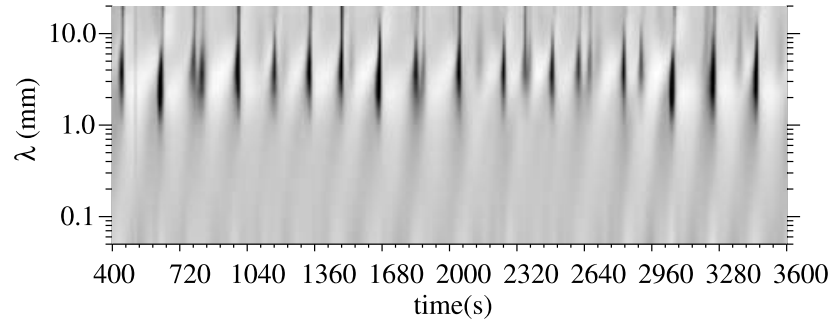

Fig. 2 Evolution of the Carlsson and Stein model millimeter spectrum with time. Negative grey scale representing excess intensity as a function of time and wavelength

with weak magnetic field like the quiet Sun internetwork, with the corresponding products expected from the simulations of Carlsson and Stein exhibits large differences. In particular, the RMS of the brightness temperature is nearly an order of magnitude larger in the model ( $800 \mathrm{~K}$ at $3 \mathrm{~mm}$ ) than in the observations $(100 \mathrm{~K})$. Another difference is the absence of longer periods in the model power spectrum. But these discrepancies do not rule out the CS models. On the one hand the model is one dimensional and hence does not predict a coherence length of the oscillations, while on the other hand we are not able to resolve individual oscillating elements due to the limited spatial resolution of the observations.

Consequently we estimated the influence of the spatial smearing on the model parameters of chromospheric dynamics and on the observed oscillatory power. Thus we confirmed that the very limited spatial resolution currently available hinders a clean separation between cells and network and typically both network and internetwork areas contribute to the recorded BIMA radiation. From the analysis of the observational data it was found that power in all frequency ranges increases significantly with improving res- 
olution. Consistency between the power predicted by the CS model and the observed power is obtained if the coherence length of oscillating elements is on the order of $1^{\prime \prime}$.

Our results are consistent with Wedemeyer-Böhm et al. (2005), who computed the millimeter wave signature resulting from the 3-D simulations of Wedemeyer et al. (2004). Although the 3-D simulations suffer from the fact that the radiative transfer of energy is computed entirely in LTE, which becomes a poor assumption at chromospheric heights, the authors believe that the chromospheric pattern and its temporal evolution is representative of the non-magnetic internetwork regions of the solar chromosphere. The simulations display a complex 3D structure of the chromospheric layers, which is highly dynamical on temporal scales of 20-25 s and on spatial scales comparable to solar granulation, which is in good agreement with the $1^{\prime \prime}$ size of oscillating elements that we deduced. According to Wedemeyer et al. (2004) the chromospheric temperature structure is characterized by a pattern of hot shock waves, which originate from convective motions, and cool gas lying between the shocks. The intensity distribution at $\mathrm{mm}$ wavelengths follows the pattern of the shocks in the chromosphere with a sub arcsecond size of the features associated with the shocks. All this complex and dynamic 3D structure can be deduced from observations at $\mathrm{mm}$ wavelengths with a sufficiently high spatial resolution of better than $1^{\prime \prime}$.

\section{Summary}

Simultaneous mm-submm observations at different wavelengths can be used for the tomography of the solar atmosphere, as radiation at the different wavelengths originates from different layers, with the average formation height increasing with wavelength. Such observations also provide a strong test of present and future models. However, observations that might be able to uncover the nature of the chromosphere should meet the following requirements:

- multiband observations in mm-submm domain (0.8$5.0 \mathrm{~mm}$ ) to address shock waves and chromospheric oscillation modes

- arcsecond spatial resolution to resolve fine structure

- temporal resolution better than a few seconds to follow its evolution in time

- FOV size of order of $1^{\prime}$

- accurate absolute calibration of the observations (Bastian 2002).

These requirements look very similar to the technical specification of the continuum observations with the Atacama Large Millimeter Array (ALMA), which represents an enormous advance over existing instrumentation operating at mm-submm wavelengths. ALMA will produce images of the highest resolution available for the foreseeable future (although the technical problem of sampling both large and small spatial scales simultaneously, required for highquality imaging of the chromosphere, will remain a challenge) and will be the most sensitive instrument operating at submm-mm wavelengths. To summarize, ALMA will be an extraordinarily powerful instrument for studying the solar chromosphere. It will finally allow the mapping of the threedimensional thermal structure of the solar chromosphere which will be a real breakthrough in solar studies.

Acknowledgements The use of BIMA for scientific research carried out at the University of Maryland is supported by NSF grant AST0028963. Solar research at the University of Maryland is supported by NSF grant ATM 99-90809 and NASA grants NAG 5-8192, NAG 5-10175, NAG 5-12860 and NAG 5-11872.

\section{References}

Ayres, T.R.: Does the Sun have a full-time COmosphere? Astrophys. J. 575, 1104-1115 (2002)

Bastian, T.S.: ALMA and the Sun. Astronomische Nachrichten 323, 271-276 (2002)

Carlsson, M., Stein, R.F.: Does a nonmagnetic solar chromosphere exist? Astrophys. J. 440, L29-L32 (1995)

Carlsson, M., Stein, R.F.: Dynamic hydrogen ionization. Astrophys. J. 572, 626-635 (2002)

Fontenla, J.M., Avrett, E.H., Loeser, R.: Energy balance in the solar transition region. III-Helium emission in hydrostatic, constantabundance models with diffusion. Astrophys. J. 406, 319-345 (1990)

Loukitcheva, M., Solanki, S.K., Carlsson, M., Stein, R.F.: Millimeter observations and chromospheric dynamics. Astron. Astrophys. 419, 747-756 (2004)

Loukitcheva, M., Solanki, S.K., White, S.: The dynamics of the solar chromosphere: comparison of model predictions with millimeterinterferometer observations. Astron. Astrophys. 456, 713-723 (2006)

Vernazza, J.E., Avrett, E.H., Loeser, R.: Structure of the solar chromosphere. III-Models of the EUV brightness components of the quiet-sun. Astrophys. J. Suppl. Ser. 45, 635-725 (1981)

Wedemeyer, S., Freytag, B., Steffen, M., Ludwig, H.-G., Holweger, H.: Numerical simulation of the three-dimensional structure and dynamics of the non-magnetic solar chromosphere. Astron. Astrophys. 414, 1121-1137 (2004)

Wedemeyer-Böhm, S., Ludwig, H.-G., Steffen, M., Freytag, B., Holweger, H.: The shock-patterned solar chromosphere in the light of ALMA. In: Favata, et al. (eds.) Proceedings of the 13th Cambridge Workshop on Cool Stars, Stellar Systems and the Sun, Hamburg, Germany, ESA SP-560, pp. 1035-1038 (2005)

White, S., Loukitcheva, M., Solanki, S.K.: High-resolution millimeterinterferometer observations of the solar chromosphere. Astron. Astrophys. 456, 697-711 (2006) 